\title{
CERVICOFACIAL EMPHYSEMA AND PNEUMOMEDIASTINUM COMPLICATING ADENOTONSILLECTOMY
}

Authors:

Adekanye A.G. ${ }^{1}$

Umana A.N. ${ }^{1}$

Akintomide A.O. ${ }^{3}$

Nakanda B. ${ }^{2}$

Mgbe R.B. ${ }^{1}$

Offiong M.E. 1

Asuquo B. ${ }^{1}$

1 Department of Otorhinolaryngology

Head and Neck Surgery

2 Department of Anesthesiology and intensive care

3 Department of Radiology

University of Calabar Teaching Hospital, Calabar, Cross River State, Nigeria.

\section{Corresponding author:}

Abiola Grace Adekanye

FWACS FICS, University of Calabar

Teaching Hospital, Calabar .

E-mail: abiolaadekanye@yahoo.com

$+23480505436065$

\section{Co-authors' email addresses:}

Aniefon Ntuen Umana:

aniefonumana@yahoo.com

Akintunde Olusijibomi Akintomide:

tundeakin@yahoo.com

Bassey Nakanda

bassey nakanda@gmail.com

Robert Bassey Mgbe:

jellyovarock@yahoo.com

Mbora Effianga Offiong:

mboraoffiong@yahoo.com

Bassey Asuquo:

dr.bassey@yahoo.com

\begin{abstract}
Subcutaneous emphysema after adenotonsillectomy is rarely encountered. One of such cases following adenotonsilectomy was seen in our institution.

We report the case of a 5-year-old girl who developed cervicofacial emphysema and pneumomediastinum following retching and vomiting 6 hours after a routine adenotonsillectomy. Radiograph of the jaws and chest revealed subcutaneous emphysema. Previously published cases showed that the cause was most likely due to air passing into subcutaneous tissue through the tonsillar fossa and superior constrictor muscle into the facial layers of the neck following a bout of coughing and straining (crying), or the use of positive pressure ventilation. The emphysema can then spread to parapharyngeal and retropharyngeal spaces with associated morbidities. Further complications may include pneumothorax and pneumomediastinum and this should be excluded.
\end{abstract}

Key words: subcutaneous emphysema; pneumomediastinum; adenotonsillectomy. 
Medical Research Archives, Vol. 5, Issue 6, June 2017

Cervicofacial emphysema and pneumomediastinum complicating adenotonsillectomy

\section{INTRODUCTION}

Removal of tonsil and adenoids, Adenotonsillectomy, is the most frequently performed surgical procedure in children worldwide, with an annual US of approximately $500,000^{1,2}$. Over the last few decades, indication has shifted from recurrent infection to airway obstruction, which is most often associated with obstructive sleep apnea (OSA) ${ }^{1-3}$. OSA is exerted by repetitive episodes of partial or complete upper airway obstruction during sleep, and is frequently associated with hypoxemia, increased inspiratory effort, and frequent awakenings. Untreated OSA is associated with cardiovascular, metabolic and neurobehavioral sequelae ${ }^{4-5}$. In school children, the most common cause of OSA is adenotonsillar hypertrophy and the main treatment option is adenotonsillectomy.

Tonsillectomy in children is relatively simple, as their tissue planes of dissection are easier to identify, unlike in older adults or elderly patients with fibrosis and hence greater chances for the occurrence of complication. Stewart AE et al in their study of 32 patients with subcutaneous emphysema and pneumomediastinum complicating tonsillectomy, reported mean age of 18.6 years (range, 2-65 years).

Generally, adenotonsillectomy is regarded as a safe procedure. However, certain risk factors may increase the incidence of perioperative respiratory complications. Known risk factors for negative adenotonsillectomy outcomes include moderate to severe OSA, obesity, young age $(<2$ years $)$ as well as comorbidities such as asthma and craniofacial malformations ${ }^{6}$ Common complications of adenotonsillectomy are: hemorrhage, pain, fever, infection and oedema of uvula. Surgical emphysema is an infrequent but potentially serious complication of tonsillectomy ${ }^{7}$. Subcutane- ous emphysema most of the time is selflimiting and can be managed conservatively. Early recognition and prompt treatment can prevent further complications ${ }^{7}$

Miman, Murat $\mathrm{C}$ et al reported a case of an 11-year-old boy who developed sudden cervical emphysema soon after he had positive pressure ventilation for partial laryngospasm after extubation ${ }^{8}$. Also, Shine Neville Patrick et al reported a case of a 7year old female that had cervicofacial emphysema and pneumomediastinum 2 hours after adenotonsillectomy and inferior turbinates electro cautery.

\section{CASE PRESENTATION}

A five-year-old girl who presented with one-year history of noisy difficult breathing associated with mouth breathing, restlessness while sleeping and apneic episodes. There were no otological symptoms. Essential finding on oropharyngeal examination was kissing tonsils. Radiograph of postnasal space showed soft tissue shadow obstructing the air column at the level of nasopharynx and oropharynx. She was initially treated conservatively without improvement. Consequently, she had adenotonsillectomy under general anesthesia with non-traumatic and uneventful endotracheal intubation. Adenoids were removed with Beckmann curette and tonsils removed by dissection method. Hemostasis was achieved by gauze tamponade. Estimated blood loss was $20 \mathrm{ml}$. At about 6 hours after the operation, she took ice cream and water orally and immediately started retching with vigorous vomiting. After the vomiting, she developed swelling involving her face, neck and chest. However, there was no associated bleeding from the nose or mouth. Physical examination revealed subcutaneous swelling of the face, neck, anterior and posterior walls of the upper part of the chest with marked crepitus on palpation (Fig.1 \&2). 
Medical Research Archives, Vol. 5, Issue 6, June 2017

Cervicofacial emphysema and pneumomediastinum complicating adenotonsillectomy

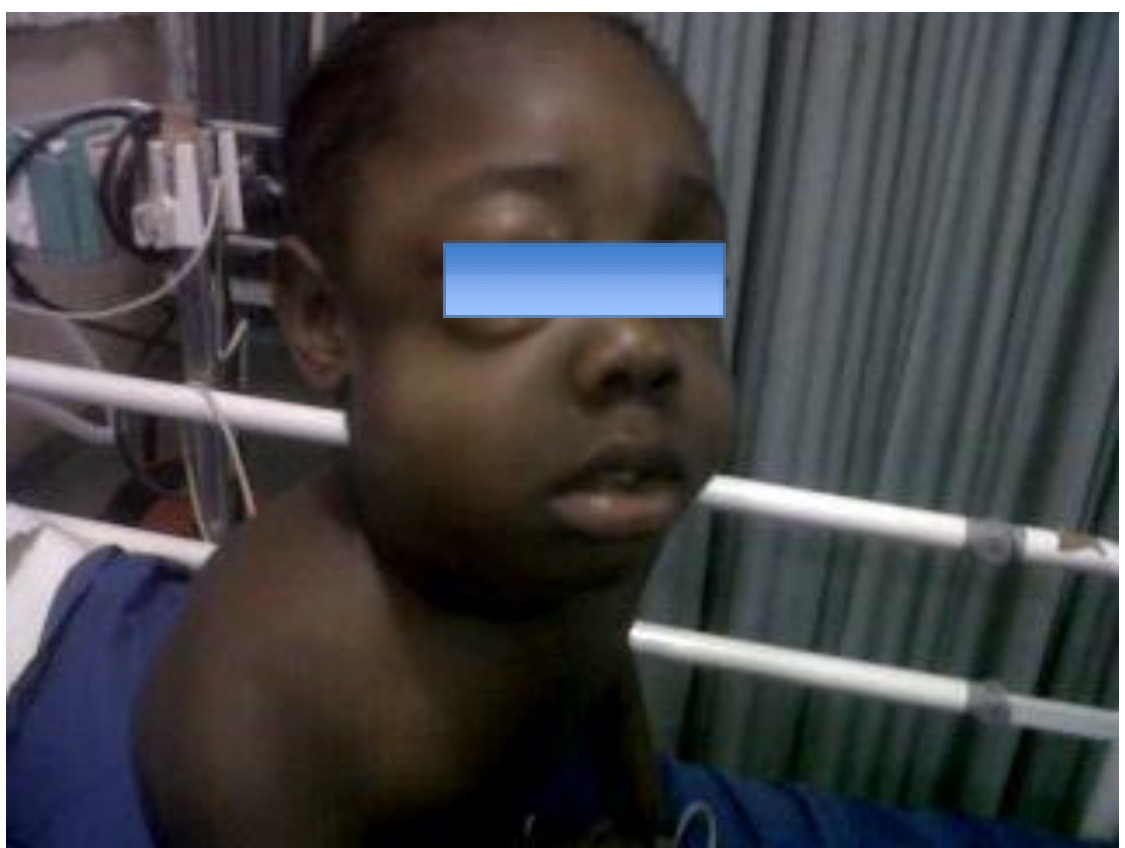

Figure 1. Subcutaneous emphysema involving Face and Chest, 6 hours post adenotonsillectomy

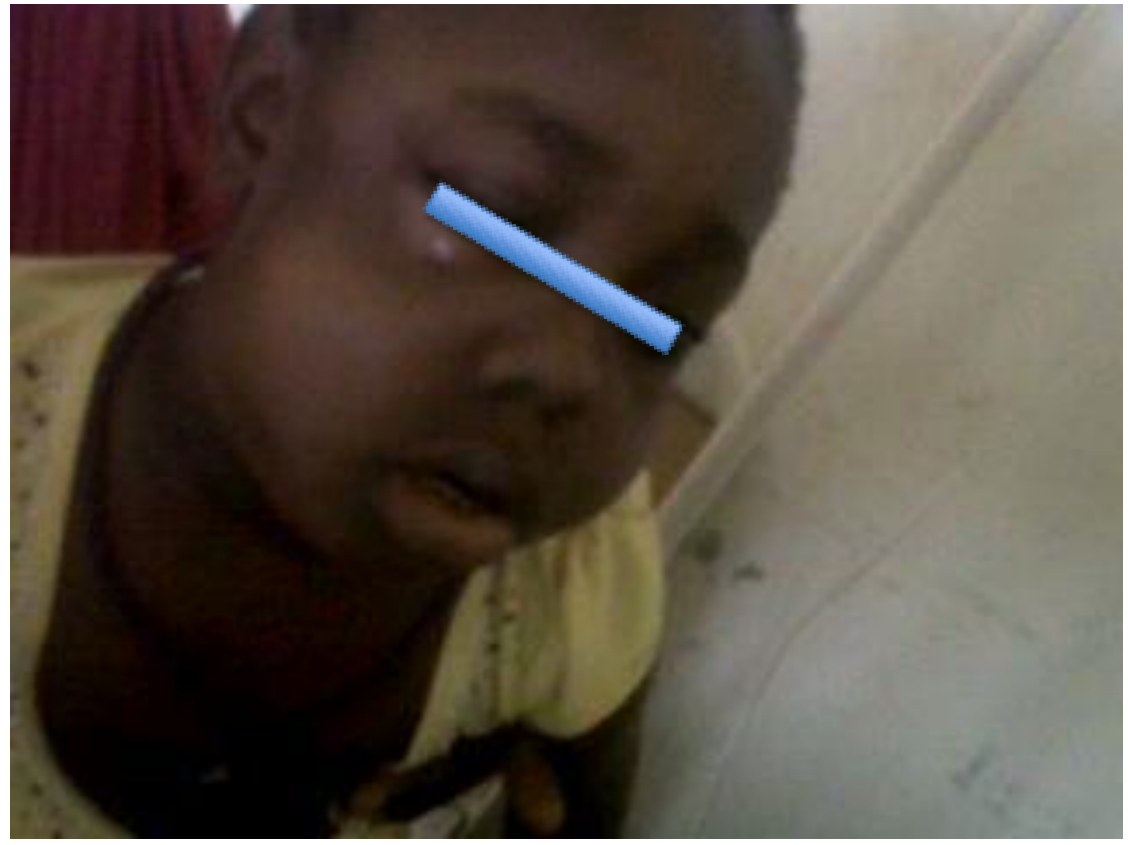

Figure 2. Subcutaneous emphysema resolving by first day post adenotonsillectomy

$\begin{array}{ccl}\begin{array}{c}\text { There was no evidence of } \\ \text { cardiovascular } \\ \text { compromise. }\end{array} \text { Plain } & \begin{array}{l}\text { revealed subcutaneous emphysema of face, } \\ \text { neck and right pneumothorax (Fig. 3\& 4). }\end{array}\end{array}$
radiographs of the jaws and neck and chest 


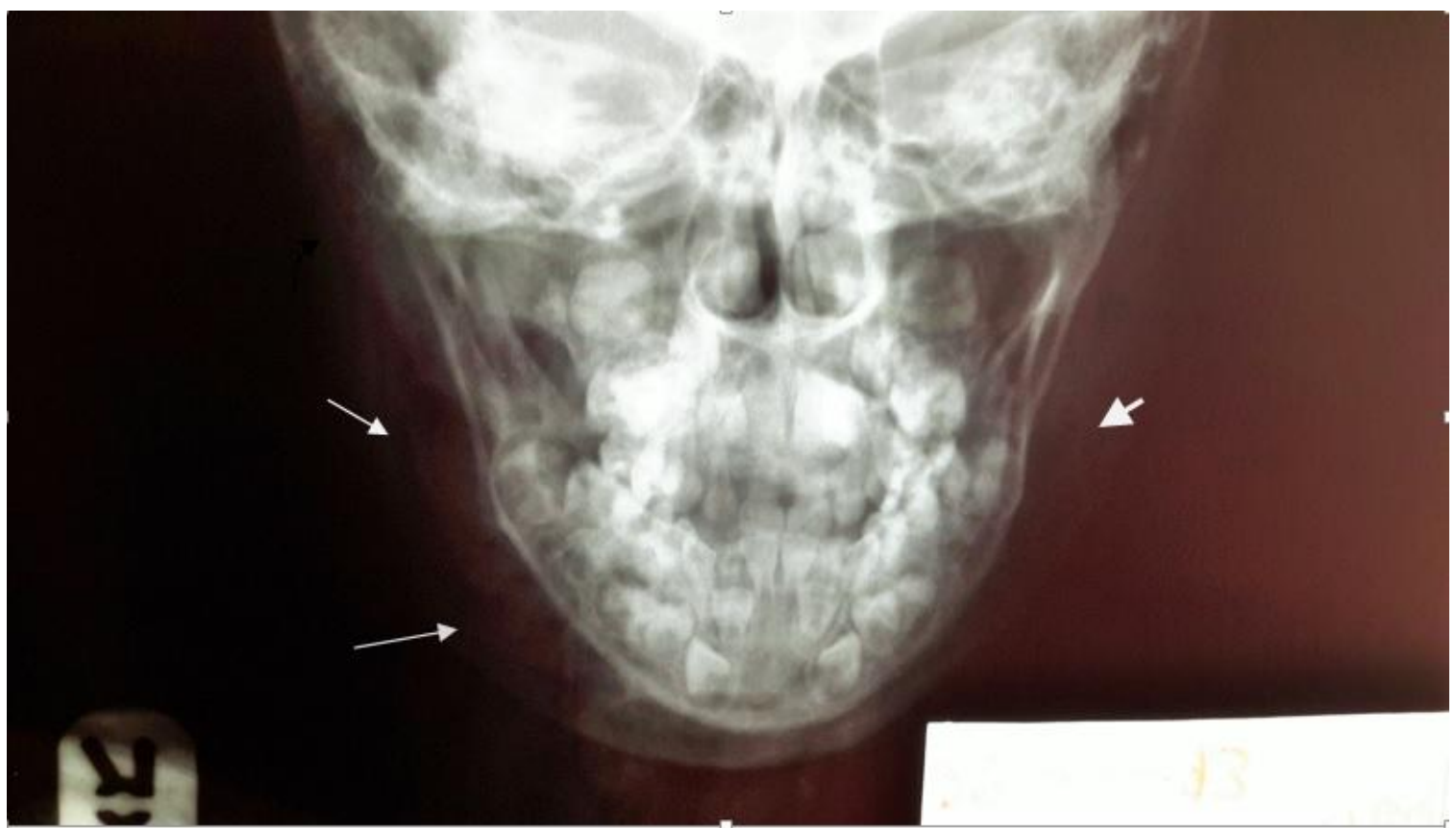

Figure 3. Jaws radiograph - Antero-posterior view shows increase in the soft tissue thickness on both sides of the jaw but more on the right. Ill defined pockets of lucencies are noted within the soft tissue: right (long arrows) and left (short arrow).

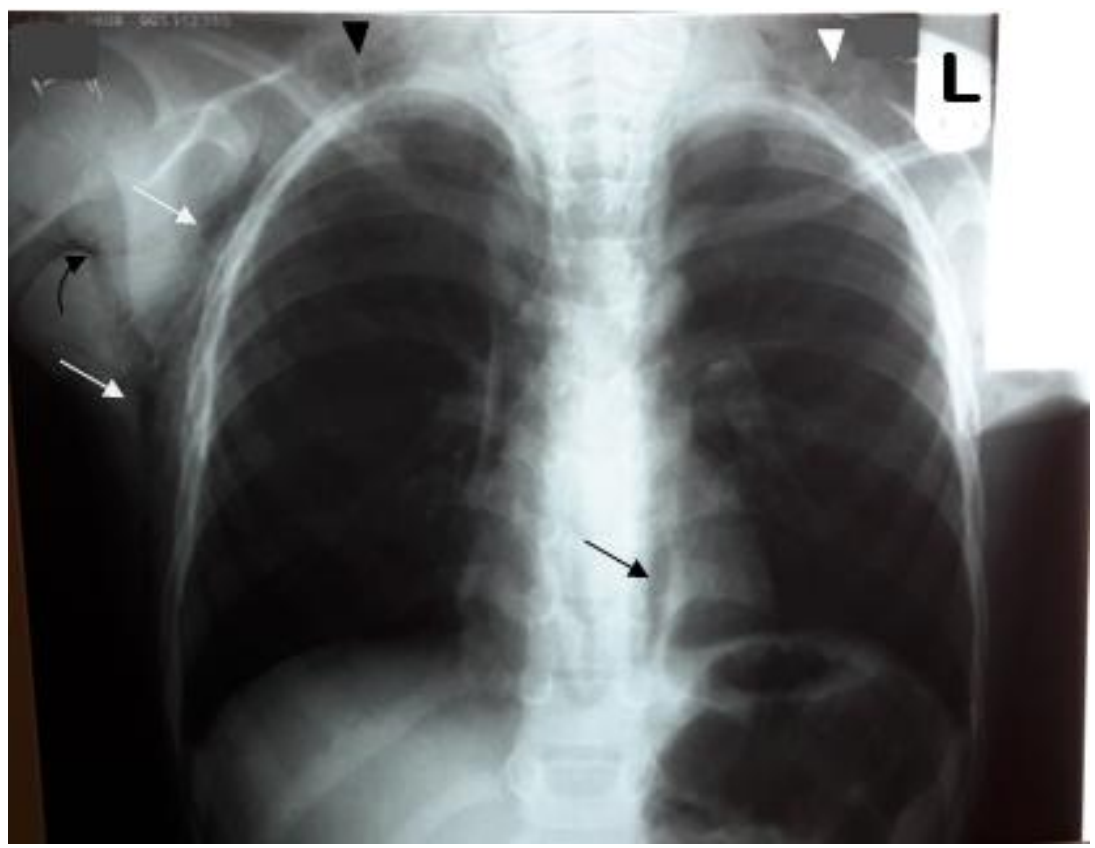

Figure 4. Chest radiograph - Posterio-anterior View shows an increase in the soft tissue thickness of the right chest wall with linear and small pockets of lucencies within (straight white arrows). Similar pockets of lucencies are noted in the right axilla (curved black arrow) and both sides of the neck; right (black arrowhead) and left (white arrowhead). Ill-defined vertically oriented lucencies suggestive of pneumomediastinum are also observed overlying the cardiac shadow centrally (straight black arrow). 
Oral intake was discontinued while she was transferred to ICU for observation (video of the patient). https://youtu.be/OmpuSU2pN7A She was successfully managed conservatively with intravenous ceftriaxone, analgesic, and hydrocortisone. Subcutaneous emphysema resolved gradually and she was discharged home on the $3^{\text {rd }}$ day after operation. She was followed up thereafter and the patient has made full recovery.

\section{Discussion}

Subcutaneous emphysema is a known entity in ENT, usually following tracheostomy, or tracheal tear. Subcutaneous emphysema and pneumomediastinum are rare complications of tonsillectomy and have been reported as far back as 1933. A literature search revealed at least 34 published cases ${ }^{9}$. Miman, Murat $\mathrm{C}$ et al reported a male child who had tonsillectomy by dissection method. He subsequently developed cervical emphysema soon after he had positive pressure ventilation for partial laryngospasm after extubation ${ }^{8}$. Shine Neville Patrick et al reported a 7-year old female that had cervicofacial emphysema and pneumomediastinum 2-hours after adenotonsillectomy and inferior turbinates electro cautery.

Our index case was the first to be encountered following adenotonsillectomy in our center.

The degree of emphysema was varied and included bilateral cases, pneumomediastinum, pneumothorax and rarely pneumoperitonium. Conservative management was successful in the vast majority of cases. In rare cases however, respiratory compromise mandated a period of ventilation by intubation or tracheostomy ${ }^{7}$. All the cases mentioned above were managed successfully.
The mechanism of subcutaneous emphysema following tonsillectomy has not been conclusively identified. A likely explanation is that inadvertent injury to the tonsillar fossae, damaging the superior constructor muscle is the cause. Such injuries to the phanygolaryngeal mucosa can be caused by surgical techniques or by injury during intubation, excessive positive or manual ventilation. This process is facilitated by events such as coughing or straining that increased upper airway pressure. Postoperative clinical examination of the case reported did not reveal a tear in the mucosa, saliva was not blood stained and no bubbles were seen in it. Intraloperatively, tonsil dissection was easy because fibrosis was not encountered in the bed. Operation time was 30 minutes and recovery from anesthesia was 10 minutes after operation.

Subcutaneous emphysema is typically associated with crepitus. Hence cervicofacial swelling with crepitus following tonsillectomy should raise suspicion of subcutaneous emphysema. Subcutaneous air can be detected with ease on a plain radiograph. It is important to check for pneumomediastinum especially if symptoms such as dyspnea, dysphagia, chest and back pain, cyanosis and Haman's sign (crepitus syndromes will systole) are present 10. Mediastinitis is a rare but potentially fatal outcome.

Our patient developed bilateral cervicofacial and pneumomediastinum after oral intake of ice cream 6 hours post adenotonsillectomy. Noticed facial swelling after retching with accompany vigorous vomiting. Facial swelling progressed and spread to the chest (anteriorly and posteriorly) bilaterally. No history suggestive of cardiorespiratory compromise. Chest radiograph confirmed cervicofacial and pneumomediastinum. 
Medical Research Archives, Vol. 5, Issue 6, June 2017

Cervicofacial emphysema and pneumomediastinum complicating adenotonsillectomy

The differential diagnosis of subcutaneous emphysema includes haemorrhage, allergic reactions and angioneurotic oedema. The existence of crepitus and X-ray findings are the characteristic of subcutaneous emphysema.

Treatment of patients with subcutaneous emphysema and pneumomediastinum based on the reported cases in the literature, involves a regular assessment of the airways and the extent of the emphysema. The aims of treatment is to prevent further air tracking within issue planes by avoiding actions that increase upper airway pressure. Such actions include coughing, vomiting, straining or vigorous activity. It is appropriate to recommend bed rest, restrict oral intake and administer cough suppressant and softener. Broad-spectrum antibiotics such as intravenous ceftriaxone may also be prescribed to prevent life threatening mediastinitis. According to the literature, oxygen therapy has been used in one previously reported case for faster absorption of subcutaneous emphysema. In most cases subcutaneous emphysema and pneumomediastinum resolved spontaneously and no deaths have been reported.

In our case report, subcutaneous emphysema and pneumomediastinum resolved gradually with close observation in the intensive care unit, restriction of oral intake, intravenous ceftriaxone, analgesic, hydrocortisone and dexamethasone. Our patient recovered completely.

\section{CONCLUSION}

Subcutaneous emphysema is said to complicate fibrotic tonsils especially in adult but can occur in children as well. Therefore, post-adenotonsillectomy/tonsillectomy patients should be monitored closely. Conservative management is effective if the condition is detected early.

\section{CONSENT}

Consent obtained from the mother of our patient to publish her daughter's case.

\section{CONFLICT OF INTERESTS}

The authors declare that there is no conflict of interests regarding the publication of this paper.

\section{ACKNOWLEDGEMENT}

We acknowledged Mrs. Grace Akpan, a senior nurse on duty who notified the doctors promptly. 
Medical Research Archives, Vol. 5, Issue 6, June 2017

Cervicofacial emphysema and pneumomediastinum complicating adenotonsillectomy

\section{REFERENCES}

1. Erickson BK, Larson DR, St SavverJL, Meverden RA, Orvidas LJ, Changes in incidence and indications of tonsillectomy and adenoidectomy 1970-2005. Otolaryngol Head Neck Surg. 2009; 6(140): 894-901.

2. Van den Akker EH, Schilder AG, Kemps YJ, Van Balen FA, Hordjk GJ, Hoes AW, Current indications for (Adeno) tonsillectomy in children: A survey in the Netherlands. Int $\mathbf{J}$ Pediatr otorhinolaryngol. 2003; 6(67): 603- 607.

3. Bhattacharyya N, Lin HW, Changes and consistencies in the epidemiology of pediatric adenotonsillar surgery 1996-2006. Otolaryngol Head Neck surg. 2010; 5 (143): 680-684.

4. Amin R, Somers VK, McConnell K, Activity-adjusted 24-hour ambulatory blood pressure and cardiac remodeling in children with sleep disordered breathing. Hypertension. 2008; 1 (51): 84-91.

5. Gozal D, Capdevila OS, KheirandishGozal L, Metabolic alterations and systemic inflammation in obstructive sleep apnea among nonobese and obese prepubertal children. Am J Respir Crit Care Med. 2008; 10 (177): 1142-1149.
6. Nafiu OO, Green GE, Walton S, Morris M, Reddy S, Tremper KK, Obesity and risk of perioperative complications in children presenting for adenotonsillectomy. Int JPediatr Otorhinolaryngol.vol. 2009; 1 (73): 89-95.

7. Hung MH, Shin PY, Yang YM, Lan JY, Fan SZ, and Jeng CS, Cervicofacial subcutaneous emphysema following tonsillectomy: implications for anaesthesiologists. Acta Anaesthesiologica Taiwanica. , 2009; 3 (47): 134-137.

8. Miman MC, Oztuan O, Durmus M, Kalcioglu MT, Gedik E, Cervical subcutaneous emphysema: an unusual complication of adenotonsillectomy. Paediatr. Anaesthisol. 200; (11): 491-493.

9. Jiang RS, Mora R, Cervicofacial surgical emphysema following tonsillectomy: a case report. Hindawi Publishing Corporation. 2014; (2014): 2.

10. Yammine NV, Alherabi A, Gerin-Lajoie J, Post- tonsillectomy subcutaneous emphysema and pneumomediastinum. J Otolaryngol. 2004;( 33):403-404. 